\title{
Patterns of clinical presentation of adult coeliac disease in a rural setting Sián Jones ${ }^{\dagger 1}$, Charles D'Souza ${ }^{\dagger 2}$ and Nadim Y Haboubi*†3
}

\author{
Address: ${ }^{1}$ Dietetics, Royal Gwent Hospital. Newport, Gwent, South Wales, UK, ${ }^{2}$ Specialist Registrar, Adult Medicine, Nevill Hall Hospital, \\ Abergavenny, Gwent, South Wales, UK and ${ }^{3}$ Consultant Physician, Adult Medicine, Nevill Hall Hospital, Abergavenny, Gwent, South Wales, UK \\ Email: Sián Jones - sian.jones2@gwent.wales.nhs.uk; Charles D'Souza - paula.harris@gwent.wales.nhs.uk; \\ Nadim Y Haboubi* - nadim.haboubi@gwent.wales.nhs.uk \\ * Corresponding author †Equal contributors
}

Published: 14 September 2006

Nutrition Journal 2006, 5:24 doi:10.1 186/1475-289/-5-24

This article is available from: http://www.nutritionj.com/content/5/I/24

(c) 2006 Jones et al; licensee BioMed Central Ltd.

This is an Open Access article distributed under the terms of the Creative Commons Attribution License (http://creativecommons.org/licenses/by/2.0), which permits unrestricted use, distribution, and reproduction in any medium, provided the original work is properly cited.
Received: 27 January 2006

Accepted: 14 September 2006

\begin{abstract}
Background: In recent years there has been increasing recognition that the pattern of presentation of coeliac disease may be changing. The classic sprue syndrome with diarrhoea and weight loss may be less common than the more subtle presentations of coeliac disease such as an isolated iron deficiency anaemia. As a result, the diagnosis of this treatable condition is often delayed or missed. Recent serologic screening tests allow non-invasive screening to identify most patients with the disease and can be applied in patients with even subtle symptoms indicative of coeliac disease. Both benign and malignant complications of coeliac disease can be avoided by early diagnosis and a strict compliance with a gluten free diet.
\end{abstract}

Aim: The aim of this study is to evaluate the trends in clinical presentation of patients diagnosed with adult coeliac disease. In addition, we studied the biochemical and serological features and the prevalence of associated conditions in patients with adult coeliac disease.

Methods: This is an observational, retrospective, cross-sectional review of the medical notes of 32 adult patients attending the specialist coeliac clinic in a district general hospital.

Results: Anaemia was the most common mode of presentation accounting for $66 \%$ of patients. Less than half of the patients had any of the classical symptoms of coeliac disease and $25 \%$ had none of the classical symptoms at presentation. Anti-gliadin antibodies, anti-endomysial antibody and anti-tissue transglutaminase showed $75 \%, 68 \%$ and $90 \%$ sensitivity respectively. In combination, serology results were $100 \%$ sensitive as screening tests for adult coeliac disease. Fifty nine percent patients had either osteoporosis or osteopenia. There were no malignant complications observed during the follow up of our patients.

Conclusion: Most adults with coeliac disease have a sub clinical form of the disease and iron deficiency anaemia may be its sole presenting symptom. Only a minority of adult coeliac disease patients present with classical mal-absorption symptoms of diarrhoea and weight loss. Patients with atypical form of disease often present initially to hospital specialists other than a gastro-enterologist. An awareness of the broad spectrum of presentations of adult coeliac disease, among doctors both in primary care and by the various hospital specialists in secondary care, is necessary to avoid delays in diagnosis. It is important to include serological screening tests for coeliac disease systematically in the evaluation of adult patients with unexplained iron deficiency anaemia or unexplained gastro-intestinal symptoms and in those who are considered to be at increased risk for coeliac disease. 


\section{Background}

Coeliac disease is an immunologically mediated enteropathy caused by a permanent intolerance to ingested gluten in genetically susceptible individuals [1-3]. Its prevalence rates in Caucasians in Europe, North and South America, Australia and the Middle East have been reported to be as high as 1 in 100 [4-6]. Typically, coeliac disease presents with symptoms of mal-absorption such as weight loss, diarrhoea, steatorrhoea, or abdominal distension. However, the symptoms of coeliac disease are diverse and it may present with a broad spectrum of clinical features such as isolated sub-clinical iron deficiency anaemia, osteoporosis, neurologic disease, non-specific abdominal symptoms, dermatitis herpetiformis or malignancies.

In recent years there has been increasing recognition that the mode of presentation of coeliac disease may be changing $[7,8]$. It often presents with symptoms not previously considered to be characteristic of the disease $[3,9,10]$. While most gastroenterologists appreciate the broader spectrum of the disease, and its increasing prevalence, it is still perceived by most general practitioners as a rare condition of childhood or infancy, presenting mainly with gastrointestinal symptoms suggestive of malabsorption [9]. There is concern that many patients with this disease are being overlooked due to failure of clinicians to consider it in the initial differential diagnosis when they present with non-classical symptoms [8]. These so-called "silent" coeliac disease patients lack diarrhoea and these non-diarrhoeal presentations now are seen more commonly than those with diarrhoea.

Early diagnosis of coeliac disease is important. In many patients with coeliac disease, the diagnosis is considered only when they present with avoidable complications such as a malignancy $[11,12]$. There is evidence that compliance with gluten free diet is protective against complications of coeliac disease, such as disorders of bone metabolism [13-15] and particularly non-Hodgkin's lymphoma [16], the most commonly associated malignant complication of coeliac disease [11].

\section{Methods}

This is a retrospective study of patients attending specialist adult coeliac disease clinic in a district general hospital in rural South Wales. Case records of all patients with adult coeliac disease who attended three consecutive clinics were reviewed. This clinic was a specialist multidisciplinary adult celiac disease clinic run by a specialist physician and a specialist dietitian in a district general hospital in rural South Wales. The diagnosis of coeliac disease was based on compatible serologic tests, small bowel biopsy, and response to a gluten free diet. Adult coeliac disease was diagnosed if the clinical presentation was after 16 years of age. The case notes were reviewed regarding the following:

1. The clinical presentation including age at diagnosis, sex distribution, source and reason for referral; presenting symptoms, the time delay between onset of symptoms and the diagnosis, family history of coeliac disease or autoimmune disorders and the presence of any associated disorders including dermatitis herpetiformis.

2. The laboratory parameters at diagnosis including haematologic tests - full blood count, red cell folate, ferritin, serum B12; serological screen (Elisa technique) anti-gliadin antibodies (IgA), endomysial antibodies (IgA), antitransglutaminase antibodies (IgA);

3. Small bowel biopsy result at diagnosis (Marsh classification);

4. Results of bone density scans - (dual energy x-ray absorptiometry: DEXA scan) - first scan following diagnosis.

If the relevant serological screening tests were not performed at the time of diagnosis, these cases were excluded from this study. Twelve (38\%) anti-gliadin antibody values, 10 (31\%) anti-endomysial antibody reports, and 4 (13\%) anti-tTG results were not available; these patients were also excluded from the study. Four (13\%) ferritin, 5 $(16 \%)$ folate and $6(19 \%)$ B12 test results were not obtainable, and these were excluded as well. In patients who were already on treatment for anaemia prior to the diagnosis of coeliac disease, haematological values before commencement of iron, B12 or folate replacement therapy were taken for analysis - as medication would have restored the subsequent values, thus creating a falsely low rate of anaemia at diagnosis, in these patients.

\section{Results}

Case records of 32 consecutive adult coeliac disease patients attending three consecutive clinics fulfilled our criteria for inclusion in this study. The mean age at diagnosis was 53.2 years with a range of 23-86 years. There were two peaks in age at diagnosis, one in the $4^{\text {th }}$ and the second, in the $6^{\text {th }}$ decade (Figure 1 ). The ratio of male: female cases was 2:3. The duration of symptoms before diagnosis ranged from 1 week to 25 years, with a mean delay in diagnosis by 71 months. There was no correlation between age at diagnosis and time elapsed between onset of symptoms and diagnosis in this study $(r=0.18, p>$ $0.05)$, thus the possibility of age related diagnostic delay is effectively ruled out. 


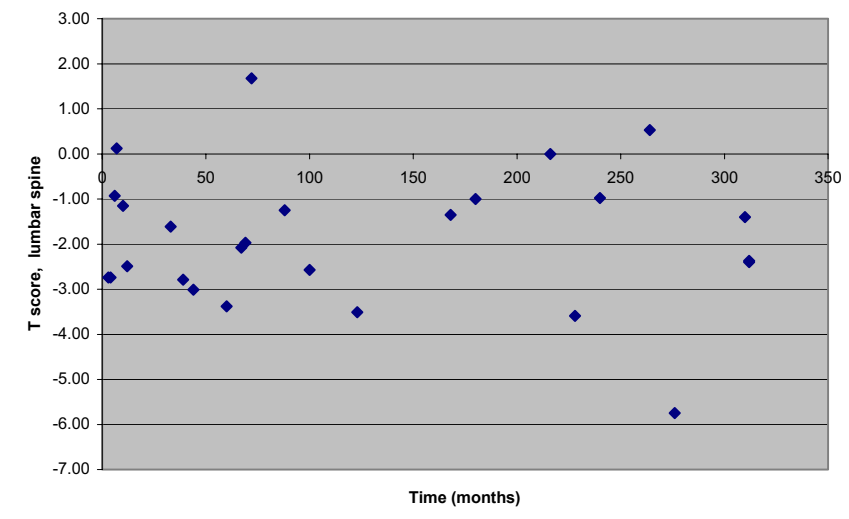

Figure I

Time on GFD.

\section{Referral source}

Thirty eight percent of patients were referred from primary care by the general practitioners; most of the remaining patients were referred from secondary care: $9 \%$ were diagnosed during inpatient evaluation following an emergency admission to hospital with acute episodes of abdominal pain, diarrhoea or vomiting. The remaining patients were referred by hospital specialists other than gastroenterologists: $22 \%$ by a haematologist, $9 \%$ by a surgeon, $22 \%$ by general hospital physicians and care of the elderly specialists (chronic anaemia), or a gynaecologist (abdominal pain), dermatologist (skin rash) and rheumatologist (iron-deficiency anaemia) (figure 2).

\section{Presenting complaints}

Anaemia was the most common mode of presentation: $68 \%$ patients had low iron stores (ferritin $<20 \mathrm{ng} / \mathrm{l}$ ) at diagnosis, 50\% had a reduced haemoglobin, 35\% had a low mean corpuscular volume (MCV) and $45 \%$ a low red
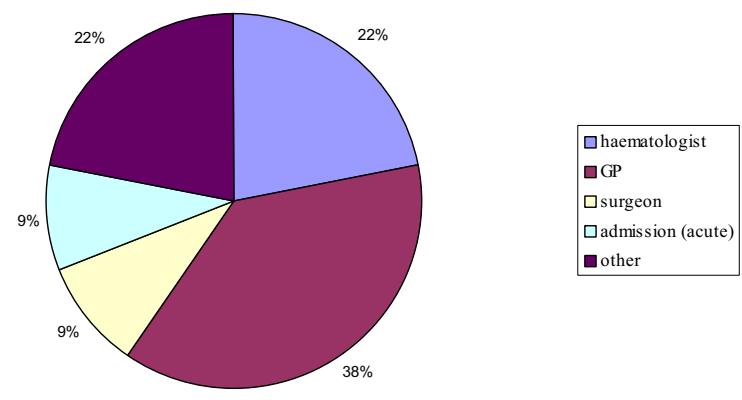

Figure 2

Source of referral. blood cell count. 13\% patients of our patients had anaemia with a high MCV at diagnosis; moreover, in some patients, a normal MCV might reflect a mixed nutritional anaemia since $57 \%$ our patients had either low folate or low B12 levels.

Less than $50 \%$ patients had at least one of the "classical" symptoms of coeliac disease; $25 \%$ (8 patients) had none of the "classical" symptoms at presentation and all these 8 subjects were found to have iron-deficiency anaemia. Only one patient (3\%) had dermatitis herpetiformis although $25 \%$ cases suffered with skin conditions "diagnosed" as follicular rash, eczema, seborrheic dermatitis, lichen planus or pruritus of unknown aetiology.

\section{Co morbidities}

Thyroid disorders were the most common disease associated with coeliac disease in this study. Amongst patients with thyroid disease, 57\% had hypothyroidism and 29\% were thyrotoxic while the remaining patient had thyroiditis. Eighty three percent of the cases with affective disorder had depression. Fifty seven percent of patients with associated arthritis had an inflammatory type of arthritis.

Fourteen percent of patients were noted to have selective IgA deficiency. The prevalence of pernicious anaemia was $9 \%$, primary biliary cirrhosis $6 \%$, and one patient had inflammatory bowel disease. Atopic disease was present in $6 \%$ of cases and one patient had sarcoidosis. No patients in this study had either type 1 diabetes mellitus or Sjögren's syndrome.

\section{Family history}

Three patients had a positive family history with a diagnosis of coeliac disease in a first degree relative. In addition, $16 \%$ of our patients gave a history of other autoimmune diseases in the family.

\section{Serological screening tests}

Seventy five percent of patients were found to have positive anti-gliadin antibodies (AGA) at diagnosis and $68 \%$ had positive endomysial antibodies (EMA) while 91\% patients were positive for EMA, AGA or both ( $95 \%$ confidence interval 79.3-102.7\%). 90\% had positive anti-tTG (95\% CI 71.4-108.5\%). However, only eight patients had had all 3 antibody screening tests performed at the time of initial diagnosis, since these tests had not been widely available until much later.

\section{Histology}

The most common finding (47\%) was partial villous atrophy; $22 \%$ had subtotal villous atrophy and $16 \%$ partialsubtotal villous atrophy, while only $13 \%$ patients demonstrated total villous atrophy (figure 3). A statistically significant correlation was found between the degree of 


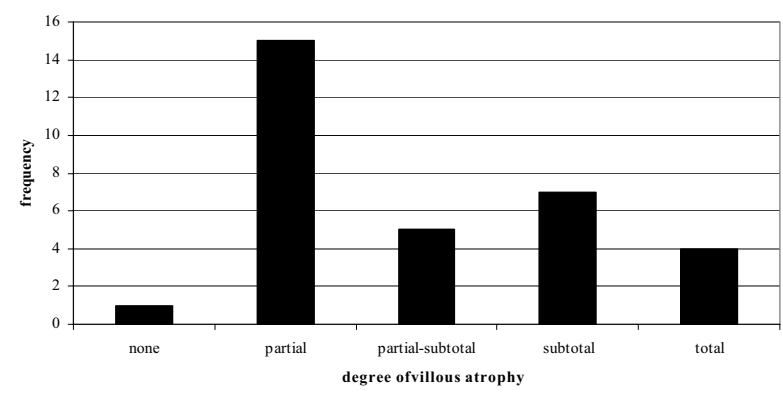

Figure 3

Findings at biopsy.

villous atrophy (no evidence of atrophy $=0$, partial v.a. $=$ 1 , partial-subtotal v.a. $=2$, subtotal v.a. $=3$, total v.a. $=4)$ and AGA levels at diagnosis $(r=0.481, p=0.013)$ (figure $4)$. The biopsy in one patient (3\%) showed only an increase in the intraepithelial lymphocytes.

\section{Complications}

Osteoporosis was the most common and also the only complication related to coeliac disease found among our 32 patients. Osteoporosis was present in 28\%, with another $31 \%$ of patients were classified as osteopenic (osteoporosis $=$ T score $<-2.5$, osteopenia $=$ T score $<-1.0$ ). DEXA scans were carried out a mean of 124.7 months after onset of symptoms (and mean 43.4 months following diagnosis). There was no correlation between $\mathrm{T}$ score and the duration of symptoms before the DEXA scan (Lspine: $r=-0.64, p=0.757$; hip: $r=-0.108, p=0.601)$, or between $\mathrm{T}$ score and the length of time elapsed following diagnosis before the DEXA scan (L-spine $r=-0.320, p=$ 0.110 ; hip $r=-0.0 .279, p=0.167)$.

There were a total four patients in our group, in whom a diagnosis of malignancy had been made on follow up: 2

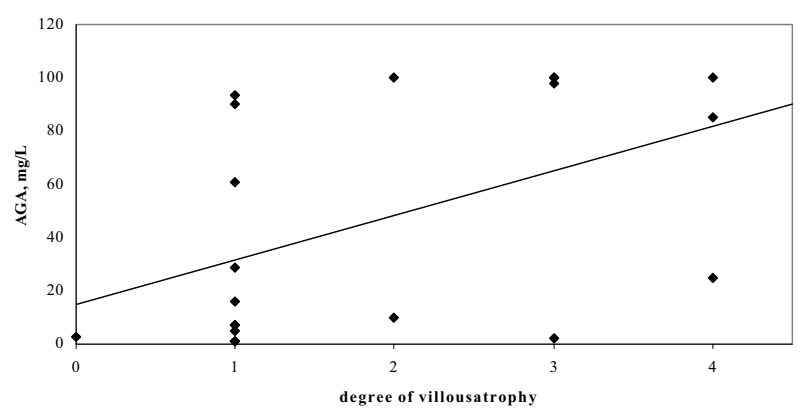

Figure 4

Association between degree of villious atrophy and AGA at diagnosis. with basal cell carcinoma, 1 each with breast cancer and melanoma. There were no cases of lymphoma, adenocarcinoma of the small intestine or oesophageal or pharyngeal carcinoma i.e. neoplasms thought to arise as a complication of coeliac disease.

\section{Discussion}

Adult coeliac disease may present with classic clinical features including weight loss, diarrhoea, and malabsorption of nutrients. However, there are reports of an increasing trend towards silent or subclinical presentations i.e. presentation with subtle symptoms not clearly related to gastrointestinal system. Nonspecific symptoms and nutritional deficiencies are especially common in older patients and as a result, the diagnosis of this treatable condition is often delayed or missed. Without active serologic screening, most cases of coeliac disease probably remain undiagnosed. The mean age at diagnosis in this study was 53.2 years with peaks in the $4^{\text {th }}$ and $6^{\text {th }}$ decades. Similar age distribution has been reported in other studies $[8,10]$. Coeliac disease is more common in women than men $[17,18]$ and the cases presented in this study concur with this, with a male: female ratio of $2: 3$. It is known that women actively seek health advice more readily than men $[7,19]$, and the high relative incidence in women may thus be a result of this behavioural pattern. It is also possible that the higher incidence in women could be due to the increased nutritional demands of women during the reproductive years [7]. This is supported by the finding that in children the ratio of boys: girls with coeliac disease approaches 1: $1[7,20]$.

Only thirty eight percent of cases in this study were referred directly from primary care by their general practitioners. Twenty two percent of patients were referred directly by a haematologist, reflecting the high incidence of anaemia among the group.

Delays in diagnosis of coeliac disease and initiation of a gluten-free diet may predispose patients to developing complications [13-15]. It has been shown that adherence to a strict gluten-free diet is protective against malignancy in coeliac disease [16]. A cohort study of 210 patients with coeliac disease who followed a gluten-free diet for a mean of 18.5 years, found that the risk of developing cancer is not significantly increased in those who followed a gluten free diet for five years or more when compared with the normal population [16]. This study found however, that those taking a reduced gluten or normal diet had an increased risk of developing cancer, especially lymphomas and cancers of the mouth, pharynx and oesophagus.

Disorders of calcium and bone metabolism were the most prevalent complications seen in coeliac disease in our study. Thirty one percent were osteopenic while $28 \%$ had 
osteoporosis and one patient had secondary hyperparathyroidism. In patients with coeliac disease, delay in diagnosis and hence delayed treatment leads to an increased risk of development of osteopenia or osteoporosis [13]. In addition, there is evidence to show that compliance with a gluten-free diet increases the bone mineral density of osteopenic coeliac patients $[14,15]$.

The most common presenting complaint in this study was iron-deficiency anaemia. Non-specific abdominal pain or discomfort, often longstanding was also a common presenting symptom. Some of our patients, who had nonspecific abdominal pain as the primary symptom, had undergone prolonged investigations elsewhere, before the diagnosis of coeliac disease was considered. Six of these 13 patients with non-specific abdominal pain had had a chronic undiagnosed anaemia for years before developing gastrointestinal symptoms which eventually led to their diagnosis.

Zipser et al. [10], in their study conducted in the USA reported fatigue to be one of the most common presenting symptom, with $82 \%$ patients complaining of this. However, fatigue was not seen as a significant symptom in our study and it was reported in only $31 \%$ of case notes. A comparison between the findings of this study and those by Zipser et al. are shown in table 1.

Although 57\% patients had either low folate or low B12 levels at the time of diagnosis, a raised MCV was seen only in a minority of patients (13\%). However, $91 \%$ of those patients with a normal MCV despite low folate or B12 levels also had low ferritin levels, reflecting their mixed deficiency anaemia, thus explaining their normocytic blood film.

Dermatitis herpetiformis was seen in only one patient in this study. However, since the diagnosis of dermatitis herpetiformis is made by skin biopsy showing pathognomonic granular IgA deposits in the papillary dermis in

Table I: Comparison between the presenting complaints of patients in this study compared to those in the study of Zipser et al. ${ }^{10}$

\begin{tabular}{lll}
\hline Complaint & Current study (\%) & Zipser et al (\%) \\
\hline Anaemia & 66 & 63 \\
Abdominal pain or discomfort & 41 & 77 \\
Diarrhoea & 38 & 52 \\
Fatigue & 31 & 82 \\
Nausea and/or vomiting & 28 & 46 \\
Weight loss & 28 & 55 \\
Depression & 19 & 46 \\
Generalised aches and pains & 19 & 42 \\
\hline
\end{tabular}

apparently unaffected skin, with increased numbers of activated T cells [21], an accurate diagnosis is unlikely to be made unless it was specifically suspected by the physician. Thus the follicular rashes, seborrhoeic dermatitis, lichen planus and pruritis recorded in the case-notes in some of our patients may actually represent undiagnosed cases of dermatitis herpetiformis.

Family history was considered as positive if any first degree relative of our patients also had a diagnosis of coeliac disease and three cases met these criteria. In family screening studies, around $10 \%$ of first degree relatives of coeliac disease patients are diagnosed with coeliac disease[8]. However, since we did not perform routine serological screening tests on the first degree relatives, we would have missed undiagnosed, sub-clinical or silent celiac disease in the family.

Sixteen percent of our patients had a first degree relative with an autoimmune disorder. The prevalence of autoimmune disorders among relatives of coeliac disease patients has been shown to be higher than among the general population. It was also shown that those relatives with an autoimmune disease were more likely to have silent, rather than symptomatic, coeliac disease [22].

The most common associated disorder seen in our patients presented here was thyroid disease (22\% patients affected). There is a well-recognised association between these two diseases $[3,11,23,24]$. One explanation for the association between coeliac disease and thyroid disorders is the coexistence of HLA molecules in both diseases [23] and HLA DR3 [23,24], HLA B8 [23] and HLA DQ8 [24] have been implicated. An alternative hypothesis is that the autoimmune reaction which occurs in the presence of gluten in coeliac disease patients promotes the development of autoimmune disorders [25]. Furthermore, it has been shown that these auto-antibodies disappear on withdrawal of gluten from the diet [26]. Many of the coeliac disease associated disorders are autoimmune in nature and $34 \%$ of patients in our study were found to have a coexisting autoimmune disorder. These results are similar to those reported by Collin et al. [27], where $28 \%$ coeliac disease patients had an associated disorder considered to be of autoimmune origin.

Arthritis and affective disorders were jointly the second most common associated disorder found among patients with coeliac disease in this study. Although, rheumatoid arthritis is frequently quoted as being coeliac-associated $[17,28,29]$, there is little evidence in the literature to support this association $[30,31]$. However, there is some evidence to support the concept of an associated enteropathic arthritis in patients with coeliac disease as has been suggested by Lubrano et al. [29] 
Nineteen percent of patients in this study had been diagnosed with an affective disorder. Fera et al. [32] suggest that affective disorders in coeliac disease are reactive, resulting from the impact of initial diagnosis and subsequent adjustment to a gluten-free diet. They recommend preventative supportive psychologic interventions in patients with a new diagnosis of coeliac disease.

The association between coeliac disease and IgA deficiency is widely recognised $[27,33,34]$. and this immunological disorder is common among coeliac disease patients. The reason for this association is unclear and it is not certain whether this is due to a common genetic factor or whether one condition predisposes to the other. The HLA alleles B8, DR3, DR7 and DQ2 are thought to be likely candidates for a genetic cause as they are prevalent in both conditions $[27,33]$. The primary concern regarding this association is the potential for a false negative serological tests for coeliac disease and hence missed diagnosis of coeliac disease as serological tests become the principal form of screening. Thus Gillett et al. [34] have argued that small bowel biopsy confirmation of diagnosis becomes essential especially in patients with selective IgA deficiency.

Although the associations between type 1 diabetes mellitus and Sjögren's syndrome with coeliac disease has been well documented $[3,8,11,20,23]$ neither of these disorders were found among patients in this study. This is most likely attributable to our small sample size.

Pernicious anaemia and primary biliary cirrhosis are among the less frequently reported disorders associated with coeliac disease; although, they were both relatively prevalent among the patients studied here $(9 \%$ and $6 \%$ respectively). Three cases of pernicious anaemia were found in our study; the association between pernicious anaemia and coeliac disease has been somewhat underreported in the literature [35].

Elevated transaminase levels is frequently found at diagnosis in patients of coeliac disease and liver cell damage, characterised by inflammation and steatosis, has been shown to resolve on a gluten-free diet [36,37]. The relationship between inflammatory bowel disease and coeliac disease is well recognised [38]. Inflammatory bowel disease is more prevalent among first degree relatives of coeliac disease patients than the normal population [39]. However, only one of our 32 patients studied here had proven ulcerative colitis, and Crohn's disease was not seen in any.

There is a possible association between coeliac disease and sarcoidosis $[23,40]$. One patient in this study had sarcoidosis which, given the rarity of this disease in this age group and in this population, may support the suggestion of association between these two disorders.

Small intestinal biopsy was diagnostic in $97 \%$ patients and was equivocal in only one patient who had an increase in intraepithelial lymphocyte reported in his duodenal biopsy. In this isolated case anti-tTG was significantly raised at $120.3 \mathrm{U} / \mathrm{ml}$ (reference range $0-10$ ). This patient had classical symptoms of abdominal discomfort and diarrhoea noted to occur following ingestion of wheat. He improved on a gluten free diet; his symptoms disappeared; and his serology normalised. In patients with positive small intestinal biopsies, it is to be noted that most patients did not a have total villous atrophy: the majority had only a partial villous atrophy. There was a statistically significant association between the histological grade and anti-gliadin antibody levels at diagnosis. This would add support to the argument of some who believe that serologic tests should now be the preferred test, to be used in the initial screening of patients thought to be at risk for coeliac disease [41].

Serological screening tests have improved case detection of coeliac disease in patients with atypical presentation. This study showed the sensitivity of EMA to be $68 \%$ and that of AGA to be $75 \%$. In combination these two antibodies gave $91 \%$ sensitivity (95\% CI 79.3-102.7\%). The sensitivity of tissue anti-tTG was $90 \%$ (95\% CI $71.4-$ $108.5 \%$ ). This demonstrates the tests to be $100 \%$ sensitive in combination, even in this study with a small sample size. Other studies have shown EMA to be $68-100 \%$ specific [42]; and when used in conjunction with AGA, a 98\% sensitivity has been reported [8]. Thus the results obtained here do not differ significantly from those reported in previous studies [43].

Serological tests may be false-negative in cases of IgA deficiency and hypogammaglobulinaemia [44]. Although there were no IgA-deficient patients in the study, one patient had hypogammaglobulinaemia. This patient however was not tested for anti-tTG at the time of diagnosis but did have positive EMA and AGA.

With the introduction of serologic tests for coeliac disease, disease variants have been recognised which present with atypical features such as fatigue, asymptomatic iron deficiency, decreased bone density and dyspepsia without the classic malabsorption syndrome. Other presentations could include isolated hypoalbuminaemia, elevated aminotransferase levels (transaminitis), microscopic colitis, symptoms of irritable bowel syndrome, recurrent aphthous stomatitis, infertility, neurologic symptoms such as peripheral neuropathy, ataxia and epilepsy with posterior cerebral calcification. The findings of this study suggest that patients presenting to their general practitioner with 
unexplained iron deficiency anaemia, unexplained fatigue or generalised abdominal pain should undergo serological testing for coeliac disease.

\section{Conclusion}

Most adults with coeliac disease have a sub-clinical form of the disease and iron deficiency anaemia may be its sole presenting symptom. Only a minority of adult coeliac disease patients present with classical mal-absorption symptoms of diarrhoea and weight loss. Patients with atypical form of disease often present initially to hospital specialists other than a gastro-enterologist. It is important to include serological screening tests for coeliac disease systematically in the evaluation of adult patients with unexplained iron deficiency anaemia or unexplained gastrointestinal symptoms and in those who are considered to be at increased risk for coeliac disease.

An awareness of the broad spectrum of presentation of patients with coeliac disease among doctors both in primary care and by the various hospital specialists in secondary care is necessary to avoid delays in diagnosis of patients with adult coeliac disease. Adherence to a strict gluten free diet is known to markedly reduce the risk of coeliac disease related complications.

\section{Competing interests}

The author(s) declare that they have no competing interests.

\section{Authors' contributions}

SJ, CDS, and NYH contributed equally to this study. All authors read and approved the final manuscript.

\section{References}

I. Marsh MN: Mucosal pathology in gluten sensitivity. In Coeliac disease Edited by: Marsh MN. Oxford: Blackwell Scientific; 1992:136-191.

2. Gee S: On the coeliac disease. St Bart Hosp Rep 1888, 24: 17-20.

3. Maki M, Collin P: Coeliac disease. Lancet 1997, 349:1755-59.

4. Csizmadia CGDS, Mearin ML, Blomberg BME, Brand R, VerlooveVanhorick SP: An iceberg of childhood disease in the Netherlands. Lancet 1999, 353:8|3-8|4.

5. Maki M, Mustalahti K, Kokkonene J: Prevalence of coeliac disease among children in Finland. N Eng J Med 2003, 348:25I 7-24.

6. West J, Logan RF, Hill PG: Seroprevalence, correlates, and characteristics of undetected coeliac disease in England. GUT 2003, 52:960-5.

7. Logan RFA, Tucker G, Rifkind EA, Heading RC, Ferguson A: Changes in clinical features of coeliac disease in adults in Edinburgh and the Lothians 1960-79. BMJ 1983, 286:95-97.

8. Feighery C: Coeliac disease. BMJ 1999, 31 19:236-9.

9. Hin H, Bird G, Fisher P, Mahy N, Jewell D: Coeliac disease in primary care: case finding study. BMJ 1999, 382:164-7.

10. Zipser RD, Patel S, Yahya KZ, Baisch DW, Monarch E: Presentations of adult celiac disease in a nationwide patient support group. Dig Dis Sci 2003, 48:76I-4.

II. Holmes GKT, Catassi C: Coeliac disease. Health Press: Oxford; 2000:174.

12. Swinson C, Slavin G, Coles EC, Booth CC: Coeliac disease and malignancy. Lancet 1983, I: I I I-I I5.

13. Bai JC, Gonsalez D, Mautalen C, Mazure R, Pedreira D, Vazuez H, Smecuol E, Siccardi A, Cataldi M, Niveloni S, Boerr LA, Maurino E:
Long-term effect of gluten restriction on bone mineral density of patients with celiac disease. Alim Pharmacol Ther 1997, II:I57-164.

14. McFarlane XA, Bhalla AK, Robertson DAF: Effect of a gluten free diet on osteopenia in adults with newly diagnosed coeliac disease. Gut 1996, 39:180-184.

15. Valdimarsson T, Lofman O, Toss G, Strom M: Reversal of osteopenia with diet in adult coeliac disease. Gut 1996, 38:322-327.

16. Holmes GKT, Prior P, Lane MR, Pope D, Allan RN: Malignancy in coeliac disease - effect of a gluten free diet. Gut 1989, 30:333-338.

17. Bottaro G, Cataldo F, Rotolo N, Spina M, Corazza GR: The clinical pattern of subclinical/silent celiac disease: an analysis on 1026 consecutive cases. Am J Gastroenterol 1999, 94:69 I-696.

18. Green PHR, Stavropoulos SN, Panagi SG, Goldstein SL, McMahon DJ, Absan $\mathrm{H}$, Neugut ATI: Characteristics of adult celiac disease in the USA: results of a national survery. Am J Gastroenterol 200I, 96:|26-|3|.

19. Hillier S, Scambler G: Women as patients and providers. In Sociology as applied to medicine 4th edition. Edited by: Scambler G. Edinburgh: WB Saunders; 1997:121-134.

20. Catassi C, Ratsch IM, Fabiani E, Rossini M, Bordicchia F, Candela F, Coppa GV, Giorgi PL: Coeliac disease in the year 2000:exploring the iceberg. Lancet 1994, 343:200-3.

21. Oxentenko AS, Murray JA: Celiac disease and dermatitis herpetiformis: the spectrum of gluten-sensitive enteropathy. Int J Dermatol 2003, 42:585-587.

22. Petaros P, Martelossi S, Tommasini A, Torre G, Caradonna M, Ventura A: Prevalence of autoimmune disorders in relatives of patients with coeliac disease. Dig Dis Sci 2002, 47:| 427 - I 43 I.

23. Collin P, Reunala T, Pukkala E, Laippala P, Keyrilainen O, Pasternack A: Coeliac disease - associated disorders and survival. Gut 1994, 35:1215-1218.

24. Hakanen M, Luotola K, Salmi J, Laippala P, Kaukinen K, Collin P: Clinical and subclinical autoimmune thyroid disease in adult celiac disease. Dig Dis Sci 200I, 46:2631-2635.

25. Ventura A, Magazzu G, Greco L: Duration of exposure to gluten and risk for autoimmune disorders in patients with celiac disease. Gastroenterol 1999, I I 7:297-303.

26. Ventura A, Neri E, Ughi C, Leopaldi A, Citta A, Not T: Glutendependent diabetes-related and thyroid-related autoantibodies in patients with celiac disease. J Pediatr 2000, 137:263-265.

27. Collin P, Maki M, Keyrilainen O, Hallstrom O, Reunala T, Pasternack A: Selective IgA deficiency and coeliac disease. Scand J Gastroentero 1992, 27:367-37I.

28. George EK, Mearin ML, Bouquet J, vonBlomberg BME, Stapel SO, vanElburg RM, deGraaf EA, HertzbergertenCate R, canSuijlekomSmit LWA, Reeser HM, Oostdijk W: Screening for coeliac disease in Dutch children with associated diseases. Acta Paediatr 1996:52-3.

29. Lubrano E, Ciacci C, Ames PRJ, Mazzacca G, Oriente P, Scarpa R: The arthritis of coeliac disease: prevalence and pattern in $\mathbf{2 0 0}$ adult patients. Brit J Rheumatol 1996, 35:1314-1318.

30. Bizzaro N, Villalta D, Tonutti E, Tampoia M, Bassetti D, Tozzoli R: Association of coeliac disease with connective tissue diseases and autoimmune diseases of the digestive tract. Autoimmun Rev 2003, 2:358-363.

31. Francis J, Carty JE, Scott BB: The prevalence of coeliac disease in rheumatoid arthritis. Eur J Gastroen Hepat 2002, I 4: I 355-1356.

32. Bizzaro N, Villalta D, Tonutti E, Tampoia M, Bassetti D, Tozzoli R: Association of coeliac disease with connective tissue diseases and autoimmune diseases of the digestive tract. Autoimmun Rev 2003, 2:358-363.

33. Cataldo F, Marino V, Bottaro G, Greco P, Ventura A: Celiac disease and selective immunoglobulin A deficiency. J Pediatr 1997, |31:306-8.

34. Gillett HR, Gillett PM, Kingstone K, Marshall T, Ferguson A: IgA deficiency and coeliac disease. J Pediatr Gastr Nutr 1997, 25:366-7.

35. Ng JP, Green ST, Lam DC, Shahriari S: Coeliac disease and pernicious anaemia. Postgrad Med J 1988, 64:889-890.

36. Kumar P, Clark M: Primary biliary cirrhosis and coeliac disease. Is there an association? Digest Liver Dis 2002, 34:248-250.

37. Bardella MT, Fraquelli M, Quatrini M, Molteni N, Bianchi P, Conte D: Prevalence of hypertransaminasemia in adult celiac patients and effect of gluten-free diet. Hepatology 1995, 22:833-836. 
38. Gillberg R, Dotevall G, Åhrén C: Chronic inflammatory bowel disease in patients with coeliac disease. Scand J Gastroentero 1982, 17:491-6.

39. Chakraborty A, Bremner A, Moore I, Beattie R: Coeliac disease and Crohn's disease: an association not to be forgotten. Hosp Med 2003, 64:684-5.

40. Douglas JG, Logan RFA, Gillon J, Grant IWB, Crompton GK: Sarcoidosis and coeliac disease: an association? Lancet 1984, ii: I3-I5.

41. Scoglio R, Di Pasquale G, Pagano G, Lucanto MC, Magazzu G, Sferlazzas C: Is intestinal biopsy always needed for diagnosis in celiac disease? Am J Gastroenterol 2002, 98:|325-|33|.

42. Sollid LM, Scott H: New tool to predict celiac disease on its way to the clinics. Gastroenterology 1998, I I5:1584- I594.

43. Dieterich W, Laag E, Schopper H, Volta U, Ferguson A, Gillett H, Riecken EO, Schuppan D: Autoantibodies to tissue transglutaminase as predictors of celiac disease. Gastroenterology 1998, I I5:|3|7-|32|.

44. Walker-Smith JA, Gaundalini S, Schmitz J, Shmerling DH, Visakorpi JK: Revised criteria for diagnosis of coeliac disease. Arch Dis Child 1990, 65:909-9|I.

Publish with Bio Med Central and every scientist can read your work free of charge

"BioMed Central will be the most significant development for disseminating the results of biomedical research in our lifetime. "

Sir Paul Nurse, Cancer Research UK

Your research papers will be:

- available free of charge to the entire biomedical community

- peer reviewed and published immediately upon acceptance

- cited in PubMed and archived on PubMed Central

- yours - you keep the copyright

Submit your manuscript here:

http://www.biomedcentral.com/info/publishing_adv.asp 